was ligated, compared with just 1 case when the gonadal vein was preserved $(3.4 \%$ of 29 procedures).

The authors suggest that patients undergoing donor or radical nephrectomy should be informed of the potential for testicular pain, and that preservation of the gonadal vein might confer a protective effect.

Original article Gjertson CK and Sundaram CP (2008) Testicular pain following laparoscopic renal surgery. J Urol 180: $2037-2040$

\section{Testosterone skin patches improve libido in postmenopausal women not receiving estrogen}

Transdermal exogenous testosterone improves libido in postmenopausal women receiving estrogen therapy. Davis et al. have now demonstrated that $300 \mu \mathrm{g}$ of testosterone a day, administered via skin patches, results in a modest but clinically important increase in sexual function in postmenopausal women with hypoactive sexual desire disorder who are not receiving estrogen.

This multicenter, double-blind study enrolled 814 women who had undergone menopause at least 12 months before enrollment and who had hypoactive sexual desire disorder. Women were randomly assigned to receive two placebo patches, one placebo patch and one $150 \mu \mathrm{g}$ testosterone patch, or two $150 \mu \mathrm{g}$ testosterone patches, daily for 52 weeks.

At week 24, the increase from baseline in the 4-week frequency of satisfying sexual episodes was significantly greater in women receiving $300 \mu \mathrm{g}$ testosterone than in patients receiving placebo (mean 2.1 episodes vs 0.7 episodes; $P<0.001)$. Compared with the placebo group, both testosterone therapy groups had a significant increase in sexual desire and a significant decrease in personal distress at week 24. The incidence of adverse effects throughout the study period was similar in all three groups, although there was a higher incidence of androgenic adverse effects, particularly increased hair growth, in women receiving $300 \mu \mathrm{g}$ testosterone. Four patients were diagnosed with breast cancer during the first 4 months of the study, one of whom had symptoms before randomization.

These findings indicate that concurrent exogenous estrogen is not required to ensure the efficacy of exogenous testosterone for hypoactive sexual desire disorder.

Original article Davis SR et al. for the APHRODITE Study Team (2008) Testosterone for low libido in postmenopausal women not taking estrogen. N Engl J Med 359: 2005-2017

\section{miR-15a and miR-16-1 microRNAs are prostate cancer suppressors}

The genes for the microRNAs miR-15a and miR-16-1 are located in the chromosomal region 13q14; deletions in this region are associated with prostate cancer progression. An Italian study has now shown that inhibition of miR-15a-miR-16-1 expression promotes prostate tumorigenesis, whereas restoration of miR-15a-miR-16-1 expression leads to tumor regression.

Bonci et al. constructed vectors that encoded antisense RNAs for miR-15a and miR-16-1; expression of the antisense RNAs led to sequestration of miR-15a and miR-16-1 and inhibition of their activity. In the noncancerous prostate cell line RWPE-1, infection with the vector conferred enhanced proliferation, survival and migration capacity, and, unlike control cells infected with a dummy virus, transformed RWPE-1 cells formed tumors in NOD-SCID mice. In addition, injection of specific antagomirs into prostates of BALB/c mice led to miR-15a-miR-16-1 downregulation and marked prostatic hyperplasia.

The researchers established that miR-15a and miR-16-1 targeted the expression of Bcl-2, Wnt3a and cyclin D1; the latter two proteins increased prostate cancer proliferation, while Wnt3a increased cell migration and, in synergy with $\mathrm{Bcl}-2$, inhibited the activity of the cytotoxic drug docetaxel. Expression of these proteins increased when miR-15a and miR-16-1 were inactivated. In mice bearing miR-15a-miR-16-1deficient prostate tumors, restoration of miR15a-miR-16-1 activity by transfection with a virus led to considerable tumor shrinkage.

The authors conclude that miR-15a and miR-16-1 are tumor suppressor genes that act by controlling the activity of Wnt3a, Bcl-2 and cyclin D1. MicroRNA therapy might have future therapeutic potential against prostate cancer.

Original article Bonci D et al. (2008) The miR-15a-miR16-1 cluster controls prostate cancer by targeting multiple oncogenic activities. Nat Med 14: 1271-1277 